Internist 2020 61:644-645

https://doi.org/10.1007/s00108-020-00800-5

Online publiziert: 24. April 2020

(c) Springer Medizin Verlag GmbH, ein Teil von Springer Nature 2020

\section{Redaktion}

C. Bokemeyer, Hamburg

M. Hallek, Köln

C. Jacobshagen, Göttingen

W. Lehmacher, Köln

U. Müller-Ladner, Bad Nauheim

$\mathrm{H}$. Wedemeyer, Essen

M. Wehling, Mannheim

\section{Originalliteratur}

Grein J, Ohmagari N, Shin D et al (2020) Compassionate use of remdesivir for patients with severe Covid-19. N Engl J Med. https://doi.org/ 10.1056/NEJMoa2007016

Seit der ersten beschriebenen Infektion mit dem „severe acute respiratory syndrome coronavirus 2" (SARS-CoV-2) im Dezember 2019 hat sich die „coronavirus disease 2019“ (COVID-19) zu einer Pandemie entwickelt, deren Symptomatik vom asymptomatischen Verlauf bis hin zu Lungenentzündung, akutem Lungen- und Multiorganversagen und Tod reicht. Um eine sinnvolle Therapiestrategie entwickeln zu können, werden unterschiedliche Medikamente „off label“ eingesetzt. Eines davon ist Remdesivir, eine Vorstufe eines Nukleotidanalogons, das virale RNA-Polymerasen inhibiert. Die Wirksamkeit von Remdesivir in der Therapie von Ebola, SARS-CoV und „Middle East respiratory syndrome coronavirus“ (MERS-CoV) konnte bereits gezeigt werden. In vitro scheint Remdesivir auch bei SARS-CoV-2 sowohl therapeutisch als auch prophylaktisch wirksam zu sein.

Ziel der im Folgenden vorgestellten Studie war es, die Wirksamkeit einer Remdesivirtherapie bei Patienten mit schwerer COVID-19-Infektion zu verifizieren.

\author{
M. Augustin ${ }^{1} \cdot$ M. Hallek' $\cdot$ S. Nitschmann ${ }^{2}$ \\ ${ }^{1}$ Klinik I für Innere Medizin, Universitätsklinikum Köln (AöR), Köln, Deutschland \\ ${ }^{2}$ Lippetal, Deutschland
}

\title{
Remdesivir bei Patienten mit schwerer COVID-19
}

\section{Zusammenfassung der Studie}

\section{S. Nitschmann \\ Lippetal, Deutschland}

\section{Studiendesign}

Nichtkontrollierte, prospektive, offene Beobachtungsstudie einer 10-tägigen Remdesivirtherapie mit einer angestrebten Nachuntersuchungszeit von 28 Tagen. Sauerstoffbedarf, unerwünschte Therapiewirkungen sowie die Laborparameter Serumkreatinin, Alaninaminotransferase (ALT) and Aspartataminotransferase (AST) an Tag 1-10 und Tag 28 sollten erhoben werden.

\section{Einschlusskriterien}

- Hospitalisierte Patienten mit schwerer SARS-CoV-2-Infektion und einer Sauerstoffsättigung $<94 \%$

- Zustimmung, keine weiteren Offlabel-Substanzen gegen COVID-19 zu bekommen

\section{Ausschlusskriterien}

- Kreatininclearance $\leq 30 \mathrm{ml} / \mathrm{min}$

- ALT und AST $\geq 5$-facher Normwert

\section{Endpunkte}

Es waren keine fixen Endpunkte geplant. Stattdessen wurde die Häufigkeit wichtiger klinischer Endpunkte quantifiziert. Hierzu gehörten:

- Sauerstoffbedarf

- Nichtinvasive Überdruckbeatmung (NIPPV), invasive Beatmung, extra- korporale Membranoxygenierung (ECMO)

- Krankenhausentlassung

- Therapienebenwirkungen

- Tod

- Laborparameter inklusive Serumkreatinin, ALT und AST an Tag 1-10 und Tag 28

\section{Methodik}

Die Patienten erhielten am ersten Tag 200 mg Remdesivir i.v., gefolgt von einer 9-tägigen $100 \mathrm{mg}$-Remdesivir-Therapie i.v. Während der Follow-up-Zeit wurden die relevanten Patientendaten (s. Endpunkte) erfasst.

\section{Ergebnisse}

Zwischen dem 25.01.2020 und dem 07.03.2020 wurden 61 Patienten in die Studie eingeschlossen. Von 7 Patienten lagen keine Nachbeobachtungsdaten vor, ein Patient erhielt eine falsche Dosie-

\section{Take home message}

Die offene Beobachtungsstudie zeigt in einer kleinen Kohorte von Patienten $(n=61)$, die aufgrund von SARS-CoV-2 schwer erkrankt waren, eine klinische Besserung unter einer Remdesivirtherapie, ohne dass die Kausalität bewiesen ist. Die vorläufigen Ergebnisse müssen daher dringend durch randomisierte und kontrollierte Studien validiert werden, um aussagekräftigere Evidenz für die Wirksamkeit und Sicherheit von Remdesivir bei COVID-19 zu erhalten. 
rung, sodass die Daten von 53 Patienten (22 aus den USA, 22 aus Europa und Kanada und 9 aus Japan) hier vorgestellt werden. Von den 53 analysierten Patienten erhielten 40 die komplette 10-tägige Remdesivirtherapie, 10 erhielten eine 5- bis 9-tägige Therapie und 3 Patienten weniger als 5 Tage Remdesivir.

Das Durchschnittsalter betrug 64 Jahre, 40 Patienten (75\%) waren Männer, 34 Patienten (64\%) wurden beatmet. Im Durchschnitt bestanden COVID-19Symptome 12 Tage, bevor die Remdesivirtherapie initiiert wurde.

In der mittleren Nachuntersuchungszeit von 18 Tagen konnte bei 36 der 53 Patienten (68\%) eine Verbesserung unter Remdesivir gezeigt werden. Eine Verbesserung konnte bei allen Patienten gezeigt werden, die keine oder lediglich eine niedrig dosierte Sauerstoffsupplementierung erhielten $(n=12)$, sowie bei 5 der 7 nichtinvasiv beatmeten Patienten. Von den 30 invasiv beatmeten Patienten konnten 17 extubiert werden und bei 3 der 4 Patienten, die eine ECMO erhielten, konnte die ECMO beendet werden; alle diese Patienten waren zum Zeitpunkt der letzten Follow-up-Untersuchung am Leben.

Insgesamt verstarben 7 der 53 Patienten (13\%), im Durchschnitt 15 Tage nach Remdesivirtherapiebeginn; 6 der 7 Patienten wurden bei Studienbeginn invasiv und einer nichtinvasiv beatmet (Hazard Ratio 2,78). Das Risiko zu versterben war bei Patienten älter als 70 Jahre (Hazard Ratio 11,34) und bei Patienten mit höherem Serumkreatininwert zu Therapiebeginn (Hazard Ratio 1,91) höher.

Therapienebenwirkungen wurden bei 32 der 53 Patienten angegeben (60\%); am häufigsten waren ein Anstieg der Leberenzyme sowie Durchfall, Hautausschlag, Nierenfunktionsstörungen und Hypotonie.

\section{Kommentar}

\section{Augustin, Prof. Dr. M. Hallek}

Klinik I für Innere Medizin, Universitätsklinikum Köln (AöR), Köln, Deutschland

Weltweit versuchen Ärzte und Forscher, eine sichere und wirksame Therapie für eine Infektion mit SARS-CoV-2 zu identifizieren. Randomisierte, kontrol- lierte Studien (RCT) dienen dazu, die Frage nach Sicherheit und Wirksamkeit neuer Therapien zu beantworten [1]. In methodisch exakt durchgeführten RCT werden die einzelnen Probanden nach Zufallsprinzip in die Interventions- und Kontrollgruppe aufgeteilt, um

- Störgrößen zu minimieren,

- eine Strukturgleichheit zu schaffen und

- eine repräsentative Antwort zur Sicherheit und Wirksamkeit einer Therapie zu bekommen.

Bei der vorliegenden Studie hingegen handelt es sich um eine nichtkontrollierte, nichtrandomisierte, offene Beobachtungsstudie. Bereits vor Studienbeginn wurden weder primäre oder sekundäre Endpunkte noch eine vorbestimmte Stichprobengröße bzw. ein Rekrutierungsstopp definiert. Diese Faktoren begrenzen erheblich den Wert der Studienergebnisse, da im Nachhinein Einfluss auf Analysezeitpunkt und Analysegewichtung genommen werden kann.

Die kleine Gesamtstudienpopulation von nur 61 Probanden ist zudem sehr heterogen: Es wurden sowohl 34 intubierte Patienten als auch 19 Patienten, die aufNormalstation mit minimalem Sauerstoff unterstützt wurden, eingeschlossen. Über 7 Probanden gibt es zum Zeitpunkt des Studieneinschlusses keine klinischen Angaben. Außerdem gibt die vorliegende Studie keinen Aufschluss darüber, wie viele Probanden aus welchem Grund im Vorfeld ausgeschlossen wurden. Weiterhin wurde Remdesivir im Median erst 12 Tage nach Auftreten der ersten Symptome verabreicht, obwohl es im Rahmen einer Infektion mit SARS-CoV-2 bereits deutlich früher zur klinischen Verschlechterung kommt [2]. Um eine Wirksamkeit von Remdesivir zu zeigen, erscheint es erheblich sinnvoller, den Wirkstoff zu einem früheren Zeitpunkt im Erkrankungsverlauf zu verabreichen.

Derzeit gibt es keine gesicherte Therapie für COVID-19. Die vorliegende Studie zeigt, dass unter Remdesivirtherapie bei $68 \%$ der Patienten mit schwerer COVID-19-Infektion eine deutliche klinische Besserung eintrat. Gleichzeitig entwickelten aber $23 \%$ der Patienten schwerwiegende Nebenwirkungen (einschließlich Multiorganversagen, septischer Schock, akute Nierenverletzung und Hypotonie). Ohne Vorliegen einer Kontrollgruppe bleibt letztlich völlig unklar, ob Remdesivir für die klinische Besserung oder die Nebenwirkungen verantwortlich ist. Daher können aus diesen Daten keinerlei wesentliche Schlussfolgerungen für die Praxis gezogen werden. Es ist verwunderlich, dass diese vorläufigen Daten so hochrangig publiziert wurden.

Um eine Aussage über die Sicherheit und Effektivität von Remdesivir in der Therapie einer SARS-CoV-2-Infektion treffen zu können, bedarf es der Durchführung von prospektiven, randomisierten, placebokontrollierten Studien (RCT).

\section{Korrespondenzadresse}

\section{Augustin}

Klinik I für Innere Medizin, Universitätsklinikum Köln (AöR)

Kerpener Str. 62, 50937 Köln, Deutschland max.augustin@uk-koeln.de

\section{Einhaltung ethischer Richtlinien}

Interessenkonflikt. M. Augustin, M. Hallek und S. Nitschmann geben an, dass kein Interessenkonflikt besteht.

Für diesen Beitrag wurden von den Autoren keine Studien an Menschen oder Tieren durchgeführt. Für die aufgeführten Studien gelten die jeweils dort angegebenen ethischen Richtlinien.

\section{Literatur}

1. Kabisch M, Ruckes C, Seibert-Grafe M, Blettner M (2011) Randomized controlled trials: part 17 of a series on evaluation of scientific publications. Dtsch Arztebl Int 108(39):663-668. https://doi. org/10.3238/arztebl.2011.0663

2. Zhou F, Yu T, Du R, Fan G, Liu Y, Liu Z, Xiang J et al (2020) Clinical course and risk factors for mortality of adult inpatients with COVID-19 in Wuhan, China: a retrospective cohort study. Lancet 395:1054-1062 